\title{
Spatial Distribution and Determinant Factors of Intimate Partner Violence Among Reproductive age Group Women in Ethiopia; Generalized Structural Equation Modeling
}

Biruk Shalmeno Tusa ( $\square$ birukshalmeno27@gmail.com )

Haramaya University College of Health and Medical Sciences https://orcid.org/0000-0003-4362-0851

Sewnet Adem Kebede

university of Gonder

Adisu Birhanu Weldesenbet

Haramaya University College of Health and Medical Sciences

\section{Research article}

Keywords: Intimate partner violence, spatial analysis, generalized structural equation modeling, Ethiopia

Posted Date: June 22nd, 2020

DOI: https://doi.org/10.21203/rs.3.rs-36036/v1

License: (c) (i) This work is licensed under a Creative Commons Attribution 4.0 International License. Read Full License

Version of Record: A version of this preprint was published at CrimRxiv on March 1st, 2022. See the published version at https://doi.org/10.21428/cb6ab371.40893c8e. 


\section{Abstract}

Background: Intimate Partner Violence (IPV) is the most serious and pervasive yet under recognized human rights violation in the world as well as in Ethiopia. The objective of this study was to find the spatial distribution of IPV and its determinant factors in Ethiopia.

Methods: Secondary data analysis was conducted among 2,687 reproductive age group women (15-49 years). The distribution of IPV across the country was observed by ArcGIS software. In SaTScan software, the Bernoulli model was fitted by Kulldorff methods to identify the purely spatial clusters of IPV. Generalized Structural Equation Model (GSEM) was used to determine factors associated with each domain of IPV (physical, emotional \& sexual violence).

Result: The spatial distribution of IPV was found to be clustered in Ethiopia with Global Moran's I 0.09 (p $<0.001)$ and the highest IPV cluster was observed in Oromia $(p<0.001)$, Somali $(p<0.001)$ and Southern Nation and Nationality and Peoples $(S N N P)(p<0.001)$ regions. Watching television and not having attitudes toward wife beating were negatively associated with physical violence. Being richest and nonsmoker were inversely associated with emotional violence. The odds of experiencing sexual violence were increased among pregnant and wife of uneducated husband/partner. Women's decision-making autonomy and husband/partner drinking alcohol have positive and negative association with all domains of IPV respectively.

Conclusions: There was a significant clustering of IPV in Ethiopia and the highest IPV cluster was observed in Oromia, Somali and SNNP regions. Being richest, watching television, not having attitudes toward wife beating, women's decision autonomy and husband's/partner's high education and nonalcohol drinker status were negatively associated with IPV. The likelihood of experiencing IPV was also increased among smoker and pregnant women. We recommend that improving the economic status of the household and power of women's decision autonomy, increasing community awareness about the consequences of IPV with particular emphasis in Oromia, Somali and SNNP regions.

\section{Background}

According to world health organization (WHO) definition intimate partner violence (IPV) is acts of physical aggression, sexual coercion, psychological abuse and controlling behaviors by current or former spouses or other intimate partners that causes physical, sexual or psychological harm (1). It is the most serious and pervasive yet under recognized human rights violation in the world $(2,3)$.

Intimate partner violence affects women's physical and mental health through direct pathways, such as injury, and indirect pathways, such as chronic health problems that arise from prolonged stress (4). It is also leading to adverse outcomes of maternal and child health (5-8). Furthermore, evidence suggests that IPV increase the risk of a woman committing suicide (9), and may also raise the risk of contracting HIV, and thus of AIDS-related death $(10,11)$. 
Intimate partner violence isn't a little issue that as it were happening in a few pockets of society, but rather is a wide-ranging public health problem of epidemic proportions, requiring urgent action. Globally, nearly one third (30\%) of all women who have been in a relationship have experienced physical and/or sexual violence by their intimate partner. This prevalence was highest in the WHO Africa, Eastern Mediterranean and South-East Asia regions (37\%) and lowest in the high-income region (23\%)(12)

In Ethiopia, the prevalence of lifetime IPV ranges from 20-78\% (13). Even though the country ratified many of international and continental agreement that promote and protect against women right, around $63 \%$ of women and $28 \%$ men agree that a husband is justified in beating his wife (14). This makes IPV not only a deep-rooted problem but also somehow acknowledged rather than challenged. In addition, there is an imbalance between men and women in institutionalized gender roles and structural power (15).

Different studies were conducted at different part of Ethiopia using a univariate analysis to determine factors associated with IPV (16-21). In this analysis only one dependent variable is allowed. However, IPV have three domains (physical, emotional and sexual violence) which need to be considered as independent variables. Furthermore the prevalence of IPV is not geographically homogenous $(13,14)$. Therefore, the present study applies spatial analysis to identify of geographic distribution IPV and generalized structural equation model (GSEM) to determine factor associated with three domains of IPV.

\section{Methods}

\section{Study design and setting}

Secondary data analysis was conducted using the Ethiopian Demography and Health Surveys (EDHS) 2016. Ethiopia is composed of 9 National Regional states: namely Tigray, Afar, Amhara, Oromia, Somali, Benishangul-Gumuz, SNNP, Gambella and Harari, and two Administrative states (Addis Ababa City administration and Dire Dawa city council). It has 68 zones, 817 districts, and 16,253 kebeles (smallest administrative units of a country) (14). The current population of country is $114,708,673$ as of Tuesday, June 2, 2020, based on Worldometer elaboration of the latest United Nations data (22).

\section{Data source and study period}

The data source for this study is secondary data, which was retrieved from the DHS program official database www.measuredhs.com, after permission was granted through online request by explaining the objective of our study. The 2016 Ethiopia Demographic and Health Survey (EDHS) is the fourth Demographic and Health Survey conducted in Ethiopia. The study period for the EDHS 2016 was from January 18,2016 , to June $27,2016(14)$.

\section{Sampling procedure, study population and sample size}

The 2016 EDHS sample was stratified and selected in two stages. In the first stage, a total of 645 Enumeration Areas (EAs) (202 in urban areas and 443 in rural areas) were selected with probability 
proportional to EA size and with independent selection in each sampling stratum.

In the second stage of selection, a fixed number of 28 households per cluster were selected with an equal probability systematic selection from the newly created household listing (14). In total, 15,683 women aged 15-49 who reported ever being married participated in the survey. For the domestic violence module, only one married woman per household was selected and 2,687 woman were selected and interviewed .The current study included women who reported ever being married and completed the IPV questionnaire (weighted sample $=2,734$ ). Latitude and longitude coordinates were also taken from selected enumeration areas (clusters). The detailed sampling procedure was presented in the full EDHS report (14).

\section{Measurements of outcome variable and operational definition}

The outcome variables with important predictors were extracted from Ethiopia Demographic and Health Surveys individual data set. In the 2016 EDHS, IPV was assessed using women's self-reported responses to questions depending on the modified Conflict Tactic Scales of Straus (23). Specifically, violence committed by the current husband/partner for currently married women and by the most recent husband/partner for formerly married women was measured by asking all ever-married women if their husband/partner ever did the following:

1. Physical violence: push you, shake you, or throw something at you; slap you; twist your arm or pull your hair; punch you with his/her fist or with something that could hurt you; kick you, drag you, or beat you up; try to choke you or burn you on purpose; or threaten or attack you with a knife, gun, or any other weapon. The respondents were categorized as having life time physical violence, if they reported at least one act since the age of 15 years (14).

2. Emotional violence: say or do something to humiliate you in front of others; threaten to hurt or harm you or someone close to you; insult you or make you feel bad about yourself The respondents were categorized as having life time emotional spousal violence, if they reported at least one act since the age of 15 years (14).

3. Sexual violence: physically force you to have sexual intercourse with him even when you did not want to; physically force you to perform any other sexual acts you did not want to; force you with threats or in any other way to perform sexual acts you did not want to. The respondents were categorized as having life time sexual violence, if they reported at least one act since the age of 15 years $(14)$.

4. Intimate partner violence (IPV): respondents were classified as having experienced lifetime IPV, if they said experiencing at least one event of physical or emotional or sexual violence since the age of 15 years $(14)$.

\section{Measurements of explanatory variable and operational definition}

Depend on different literature review (24-37); variables related to women, husband/ partner and family were included in this analysis Fig. 1. 
1. Attitudes toward wife beating: It was measured based on the following five questions that women were asked about whether situations of hitting or beating a wife is justifiable: if she goes out without telling him; neglects their children; argues with him; refuses to have sex with him; and burns the food. If they said 'yes' to any one of the above questions, they were classified as having attitude towards wife beating.

2. Women's decision-making autonomy: Categorized as 'yes' if she was involved in all decisions regarding her own health care, major household purchases and visits to her family or relatives

\section{Data processing}

The analysis was done using STATA 14, ArcGIS 10.3 and SaTScan 9.6 software's. Before any statistical analysis, the data was weighted using sampling weight (weight for domestic violence), primary sampling unit, and strata to restore the representativeness of the survey and to tell the STATA to consider the sampling design when calculating standard errors to get reliable statistical estimates. Descriptive statistics and summary statistics were showed using text and tables.

\section{Spatial analysis}

\section{Spatial autocorrelation analysis}

To check whether IPV were spread or cluster or randomly distributed, spatial autocorrelation (Global Moran's I) statistic measure was used. Moran's I values close to-1 indicated disease/event dispersed, whereas Moran's I values close to +1 indicated disease/event clustered, and disease/event distributed randomly if Moran's I value was zero. A statistically significant Moran's I $(p<0.05)$ led to the rejection of the null hypothesis (IPV is randomly distributed) and indicated the presence of spatial autocorrelation. This analysis was done using ArcGIS software.

\section{Spatial scan statistical analysis}

Statistically significant spatial clusters of IPV among reproductive age group women were identified by spatial scan statistical analysis using Kuldorff's SaTScan software. The maximum cluster size was set at $50 \%$ of the population at risk. Bernoulli model was fitted by considering women who did not experience life time IPV taken as controls and those women who experience life time IPV were taken as cases represented by a $0 / 1$ variable. The number of cases in each location had Bernoulli distribution and the model required data with or without life time IPV. A Likelihood ratio test statistic was used to determine whether the number of observed life time IPV cases within the potential cluster was significantly higher than the expected or not. Primary and secondary clusters were identified using p-values and likelihood ratio tests based on the 999 Monte Carlo replications.

\section{Model building for Generalized Structural Equation model}

Generalized Structural Equation Model (GSEM) was used to determine factors associated with each domain of IPV (physical, emotional and social violence). Each domain of IPV were binary variable that 
was analyzed with Bernoulli family and a logit link function.

The analysis was started with a hypothesized model in Fig. 1. Modifications were taken iteratively by adding a path link. At the end, an over identified model with minimum information criteria was retained. A final model was selected based on statistical significance of path coefficient, the theoretical meaningfulness of the relationship and minimum information criteria. Statistically significant effects were considered for $\mathrm{P}<0.05$ at Confidence interval of $95 \%$.

\section{Result}

\section{Characteristics of study population}

A data of 2,687 reproductive age group women were included in the final analysis. Among these study participants, more than one third of them (39.62\%) were from Oromia region, more than two third $(68.87 \%)$ of them were not currently working and 1,107 (41.20\%) are Muslim. More than third quarters $(78.5 \%)$ of the respondents were not watching television at all. Around $11.1 \%$ of the respondents were pregnant. More than two third of the women had decision making autonomy $(69.00 \%)$ and have attitudes towards wife beating (68.63). Regarding husband / partner of respondents, $44.80 \%$ of them did not have any formal education and $30.23 \%$ of them drunk alcohol. 
Table 1

Socio-demographic characteristics of respondents in Ethiopia from January 18 to June $27,2016(\mathrm{~N}=2,687)$

\begin{tabular}{|c|c|c|}
\hline Variables & Weighted frequency & Percent\% \\
\hline \multicolumn{3}{|l|}{ Age } \\
\hline $15-19$ & 137 & 5.02 \\
\hline $20-24$ & 453 & 16.56 \\
\hline $25-29$ & 686 & 25.09 \\
\hline $30-34$ & 577 & 21.11 \\
\hline $35-39$ & 464 & 16.97 \\
\hline $40-44$ & 283 & 10.36 \\
\hline $45-49$ & 134 & 4.88 \\
\hline \multicolumn{3}{|l|}{ Residence } \\
\hline Urban & 387 & 14.15 \\
\hline Rural & 2,347 & 85.85 \\
\hline \multicolumn{3}{|l|}{ Region } \\
\hline Tigray & 159 & 5.80 \\
\hline Afar & 16 & 0.57 \\
\hline Amhara & 687 & 25.14 \\
\hline Oromia & 1,083 & 39.62 \\
\hline Somali & 62 & 2.28 \\
\hline Benishangul & 30 & 1.09 \\
\hline SNNP & 596 & 21.79 \\
\hline Gambella & 6 & 0.21 \\
\hline Harari & 5 & 0.18 \\
\hline Addis Ababa & 78 & 2.86 \\
\hline Dire Dawa & 12 & 0.45 \\
\hline \multicolumn{3}{|l|}{ Religion } \\
\hline Orthodox & 1,032 & 38.41 \\
\hline
\end{tabular}

SNNP: Southern Nation and Nationality and Peoples 


\begin{tabular}{|c|c|c|}
\hline Variables & Weighted frequency & Percent\% \\
\hline Muslim & 1,107 & 41.20 \\
\hline Protestant & 501 & 18.65 \\
\hline Traditional & 19 & 0.71 \\
\hline Catholic & 14 & 0.52 \\
\hline Other & 14 & 0.52 \\
\hline \multicolumn{3}{|l|}{ Educational status } \\
\hline No education & 1,707 & 62.43 \\
\hline Primary & 762 & 27.86 \\
\hline Secondary & 168 & 6.16 \\
\hline Higher & 97 & 3.55 \\
\hline \multicolumn{3}{|l|}{ Currently working } \\
\hline Yes & 851 & 31.13 \\
\hline No & 1,883 & 68.87 \\
\hline \multicolumn{3}{|l|}{ Smokes cigarettes } \\
\hline Yes & 2,718 & 99.41 \\
\hline No & 16 & 0.59 \\
\hline \multicolumn{3}{|c|}{ Attitudes toward wife beating } \\
\hline Have & 1,876 & 68.63 \\
\hline Have no & 858 & 31.37 \\
\hline \multicolumn{3}{|c|}{ Frequency of listening to radio } \\
\hline Not at all & 1,949 & 71.30 \\
\hline Less than once a week & 387 & 14.16 \\
\hline At least once a week & 398 & 14.54 \\
\hline \multicolumn{3}{|c|}{ Frequency of watching television } \\
\hline Not at all & 2,156 & 78.85 \\
\hline Less than once a week & 275 & 10.07 \\
\hline At least once a week & 303 & 11.08 \\
\hline
\end{tabular}

SNNP: Southern Nation and Nationality and Peoples 


\begin{tabular}{|c|c|c|}
\hline Variables & Weighted frequency & Percent\% \\
\hline \multicolumn{3}{|c|}{ Currently pregnant } \\
\hline Yes & 301 & 11.01 \\
\hline No or unsure & 2,433 & 88.99 \\
\hline \multicolumn{3}{|c|}{ Household wealth index } \\
\hline Poorest & 483 & 17.68 \\
\hline Poorer & 584 & 21.37 \\
\hline Middle & 574 & 20.98 \\
\hline Richer & 544 & 19.91 \\
\hline Richest & 549 & 20.06 \\
\hline \multicolumn{3}{|c|}{ Women's decision-making autonomy } \\
\hline Yes & 1,890 & 69.00 \\
\hline No & 844 & 31.00 \\
\hline \multicolumn{3}{|c|}{ Husband/partner age } \\
\hline $15-19$ & 13 & 0.47 \\
\hline $20-24$ & 122 & 4.47 \\
\hline $25-29$ & 434 & 15.88 \\
\hline $30-34$ & 565 & 20.65 \\
\hline $35-39$ & 473 & 17.29 \\
\hline $40-44$ & 466 & 17.06 \\
\hline $45-49$ & 344 & 12.59 \\
\hline $50-54$ & 204 & 7.45 \\
\hline$>55$ & 114 & 4.13 \\
\hline \multicolumn{3}{|c|}{ Husband/partner's education level } \\
\hline No education & 1,225 & 44.80 \\
\hline Primary & 1,075 & 39.34 \\
\hline Secondary & 229 & 8.37 \\
\hline Higher & 190 & 6.94 \\
\hline
\end{tabular}

SNNP: Southern Nation and Nationality and Peoples 


\begin{tabular}{|lll|}
\hline Variables & Weighted frequency & Percent\% \\
\hline Don't know & 15 & 0.55 \\
\hline Husband/partner's working status & & \\
\hline Yes & 2,583 & 94.49 \\
\hline No & 151 & 5.51 \\
\hline Husband/partner drinks alcohol & & \\
Yes & 826 & 30.23 \\
\hline No & 1,908 & 69.77 \\
\hline SNNP: Southern Nation and Nationality and Peoples & \\
\hline
\end{tabular}

\section{Spatial distribution of intimate partner violence}

The spatial distribution of IPV was found to be clustered in Ethiopia with Global Moran's I 0.09 ( $p<$ 0.001 ). Given the $z$-score of 5.45 , there is less than $1 \%$ likelihood that this clustered pattern could be the result of random chance. The bright red and blue color to the end tails shows an increased significance level (Fig. 2). Spatial clustering of IPV was found at regional levels. The highest IPV was spatially clustered in Oromia, SNNPR,Somalia and Amhara (Fig. 3).

\section{Spatial SaTScan analysis of intimate partner violence (Bernoulli based model)}

Spatial scan statistics detected a total of high and modest preforming spatial cluster of IPV. Among these, 10 clusters were high performing cluster $(L L R=40.67, R R=2.46$, P-value $<0.001)$ and 4 clusters were lowest performing cluster $(L L R=13.19, R R=1.98$, P-value $<0.001)$. The highest performing clusters of IPV were identified in Oromia, Somali and SNNPR Table 2. The bright red colors (rings) indicate that the most statistically significant spatial windows of IPV. There was high IPV within the cluster than outside the cluster (Fig. 4).

Table 2

Significant spatial clusters with high rate intimate partner violence among women in Ethiopia, 2016

\begin{tabular}{|c|c|c|c|c|c|c|c|}
\hline Cluster & $\begin{array}{l}\text { Enumeration area } \\
\text { (cluster) identified }\end{array}$ & $\begin{array}{l}\text { Coordinate } \\
\text { (radius) }\end{array}$ & Population & Case & RR & LLR & $\begin{array}{l}\mathrm{P} \text { - } \\
\text { value }\end{array}$ \\
\hline 1 & $\begin{array}{l}377,394,422,7,34, \\
289,480,398,316, \\
601\end{array}$ & $\begin{array}{l}(5.203234 \mathrm{~N}, \\
40.019732 \mathrm{E}) / \\
187.83 \mathrm{~km}\end{array}$ & 99 & 75 & 2.46 & 40.67 & $\begin{array}{l}< \\
0.001\end{array}$ \\
\hline 2 & $447,486,227,432$ & $\begin{array}{l}(7.527086 \mathrm{~N}, \\
36.970948 \mathrm{E}) / \\
45.13 \mathrm{~km}\end{array}$ & 67 & 42 & 1.98 & 13.19 & $\begin{array}{l}<.001 \\
0.001\end{array}$ \\
\hline
\end{tabular}




\section{Determinant factors of intimate partner violence}

The final model for determinant factors IPV is shown in Fig. 5 and Table 3. This model had included twelve exogenous variables (age, religion, region, wealth index, frequency of watching television, smokes cigarettes, currently pregnant, attitudes toward wife beating Women's decision-making autonomy, husband/partner age, husband/partner's education level, husband/partner drinks alcohol) and three endogenous (physical, emotional and sexual violence). All path coefficients were statistically significant at an alpha level of 0.05 .

Numerous variables, namely residence, educational status, currently working, frequency of listening to radio, husband/partner's education level, husband/partner's working status were excluded from the final model as their effect were not statistically significant at an alpha level of 0.05 .

The likelihood of experiencing physical violence was decreased by $46 \%$ among women with age $15-19$ than women with age 25-29. Traditional religion followers were 3.64 times more likely to be sexually violated than Muslim religion followers. The odds of emotional violence were decreased by $44 \%$ among richest than the poorest.

The odds of experiencing physical violence was decreased in Tigray, Afar, Amhara, Gambella, Benishangul, SNNP and Somali as compare with Oromia region. In addition, the likelihood of emotional violence was decreased Afar, Amhara and Somali when it compares with Oromia region. Furthermore, the chance of sexual violence was decreased among women from Tigray, Afar, Amhara, Somali, Benishangul, SNNP, Harari, Addis Ababa, Dire Dawa and Somali compared to those from Oromia region.

[F_Television: frequency of watching television, Hub_Age: Husband/partner age, Women's-DMA: Women's decision-making autonomy, Hub_Drinksalchol: Husband/partner drinks alcohol, Hub_Edu: Husband/partner's education level, HH_Wealth: Household wealth index, Phy_violence: Physical violence, Emo_violence: Emotional violence

The likelihood of experiencing physical violence were decreased by $36 \%$ and $42 \%$ among women who were watching television less than once a week and at least once a week as compared with those were not watching television at all respectively. Smoker women were 2.59 times more likely to be emotionally violated than their counterpart. Pregnant women were 1.62 times more probable to be sexually violated than non-pregnant women.

Women who have attitudes toward wife beating were 1.32 times more likely to be physically violated than their counterpart. The chances of physical, emotional and sexual violence were decreased by $28 \%, 30 \%$ and $35 \%$ among women who had decision making autonomy than their counterparts respectively.

Husband /partner's age, educational level and alcohol drinking status were significant husband / partner related factors that determine IPV. The likelihoods of emotional violence were decreased by $49 \%$ and $38 \%$ among husband/partner's age with 20-24 and 25-29 as compared with husband/partner's age with 3034 respectively. The odd of sexual violence was decreased by $61 \%$ among higher educated 
husband/partner than uneducated. Drinker husband / partner were 3.12, 2.44 and 2.58 times more likely to physically, emotionally and sexually violate his wife/ partner than their counterparts respectively. 
Table 3

Determinant factors of intimate partner violence among reproductive age group women in Ethiopia, 2016

\begin{tabular}{|c|c|c|c|c|c|c|}
\hline \multirow[t]{2}{*}{ Variables } & \multicolumn{2}{|c|}{ Physical violence } & \multicolumn{2}{|c|}{$\begin{array}{l}\text { Emotional } \\
\text { Violence }\end{array}$} & \multicolumn{2}{|c|}{ Sexual Violence } \\
\hline & AOR & $95 \% \mathrm{Cl}$ & AOR & $95 \% \mathrm{Cl}$ & AOR & $95 \% \mathrm{Cl}$ \\
\hline \multicolumn{7}{|l|}{ Age } \\
\hline $25-29$ & Ref & Ref & Ref & Ref & Ref & Ref \\
\hline $15-19$ & 0.54 & $\begin{array}{l}(0.31- \\
0.93)\end{array}$ & - & - & - & - \\
\hline $20-24$ & 0.97 & $\begin{array}{l}(0.71- \\
1.32)\end{array}$ & - & - & - & - \\
\hline $30-34$ & 1.06 & $\begin{array}{l}(0.80- \\
1.43)\end{array}$ & - & - & - & - \\
\hline $35-39$ & 1.30 & $\begin{array}{l}(0.95- \\
1.77)\end{array}$ & - & - & - & - \\
\hline $40-44$ & 1.06 & $\begin{array}{l}(0.71- \\
1.57)\end{array}$ & - & - & - & - \\
\hline $45-49$ & 1.04 & $\begin{array}{l}(0.62- \\
1.76)\end{array}$ & - & - & - & - \\
\hline \multicolumn{7}{|l|}{ Religion } \\
\hline Muslim & Ref & Ref & Ref & Ref & Ref & Ref \\
\hline Orthodox & - & - & - & - & 0.88 & $\begin{array}{l}(0.54- \\
1.42)\end{array}$ \\
\hline Protestant & - & - & - & - & 0.85 & $\begin{array}{l}(0.51- \\
1.41)\end{array}$ \\
\hline Traditional & - & - & - & - & 3.64 & $\begin{array}{l}(1.19- \\
11.15)\end{array}$ \\
\hline Catholic & - & - & - & - & 0.66 & $\begin{array}{l}(0.08- \\
5.44)\end{array}$ \\
\hline \multicolumn{7}{|l|}{ Region } \\
\hline Oromia & Ref & Ref & Ref & Ref & Ref & Ref \\
\hline Tigray & 0.22 & $\begin{array}{l}(0.13- \\
0.34)\end{array}$ & 0.71 & $\begin{array}{l}(0.48- \\
1.06)\end{array}$ & 0.52 & $\begin{array}{l}(0.29- \\
0.94)\end{array}$ \\
\hline Afar & 0.36 & $\begin{array}{l}(0.21- \\
0.62)\end{array}$ & 0.46 & $\begin{array}{l}(0.26- \\
0.81)\end{array}$ & 0.18 & $\begin{array}{l}(0.06- \\
0.51)\end{array}$ \\
\hline
\end{tabular}

SNNP: Southern Nation and Nationality and Peoples 


\begin{tabular}{|c|c|c|c|c|c|c|}
\hline \multirow[t]{2}{*}{ Variables } & \multicolumn{2}{|c|}{ Physical violence } & \multicolumn{2}{|c|}{$\begin{array}{l}\text { Emotional } \\
\text { Violence }\end{array}$} & \multicolumn{2}{|c|}{ Sexual Violence } \\
\hline & AOR & $95 \% \mathrm{Cl}$ & AOR & $95 \% \mathrm{Cl}$ & AOR & $95 \% \mathrm{Cl}$ \\
\hline Amhara & 0.36 & $\begin{array}{l}(0.25- \\
0.52)\end{array}$ & 0.57 & $\begin{array}{l}(0.40- \\
0.82)\end{array}$ & 0.42 & $\begin{array}{l}(0.25- \\
0.71)\end{array}$ \\
\hline Somali & 0.16 & $\begin{array}{l}(0.09- \\
0.31)\end{array}$ & 0.21 & $\begin{array}{l}(0.11- \\
0.38)\end{array}$ & 0.03 & $\begin{array}{l}(0.01- \\
0.21)\end{array}$ \\
\hline Benishangul & 0.50 & $\begin{array}{l}(0.34- \\
0.74)\end{array}$ & 0.85 & $\begin{array}{l}(0.58- \\
1.24)\end{array}$ & 0.36 & $\begin{array}{l}(0.20- \\
0.63)\end{array}$ \\
\hline SNNP & 0.53 & $\begin{array}{l}(0.38- \\
0.75)\end{array}$ & 0.80 & $\begin{array}{l}(0.57- \\
1.12)\end{array}$ & 0.40 & $\begin{array}{l}(0.23- \\
0.69)\end{array}$ \\
\hline Gambella & 0.67 & $\begin{array}{l}(0.43- \\
1.06)\end{array}$ & 0.97 & $\begin{array}{l}(0.62- \\
1.53)\end{array}$ & 0.50 & $\begin{array}{l}(0.25- \\
1.01)\end{array}$ \\
\hline Harari & 1.20 & $\begin{array}{l}(0.76- \\
1.89)\end{array}$ & 1.99 & $\begin{array}{l}(1.28- \\
3.09)\end{array}$ & 0.42 & $\begin{array}{l}(0.18- \\
0.97)\end{array}$ \\
\hline Addis Ababa & 0.60 & $\begin{array}{l}(0.35- \\
1.05)\end{array}$ & 0.79 & $\begin{array}{l}(0.46- \\
1.36)\end{array}$ & 0.21 & $\begin{array}{l}(0.08- \\
0.56)\end{array}$ \\
\hline Dire Dawa & 0.73 & $\begin{array}{l}(0.46- \\
1.16)\end{array}$ & 1.12 & $\begin{array}{l}(0.71- \\
1.78)\end{array}$ & 0.37 & $\begin{array}{l}(0.17- \\
0.80)\end{array}$ \\
\hline \multicolumn{7}{|l|}{ Wealth index } \\
\hline Poorest & Ref & Ref & Ref & Ref & Ref & Ref \\
\hline Poorer & - & - & 0.91 & $\begin{array}{l}(0.67- \\
1.23)\end{array}$ & - & - \\
\hline Middle & - & - & 1.01 & $\begin{array}{l}(0.73- \\
1.38)\end{array}$ & - & - \\
\hline Richer & - & - & 0.87 & $\begin{array}{l}(0.63- \\
1.21)\end{array}$ & - & - \\
\hline Richest & - & - & 0.56 & $\begin{array}{l}(0.40- \\
0.78)\end{array}$ & - & - \\
\hline \multicolumn{7}{|c|}{ Frequency of watching television } \\
\hline Not at all & Ref & Ref & Ref & Ref & Ref & Ref \\
\hline Less than once a week & 0.64 & $\begin{array}{l}(0.45- \\
0.93)\end{array}$ & - & - & - & - \\
\hline At least once a week & 0.58 & $\begin{array}{l}(0.41- \\
0.83)\end{array}$ & - & - & - & - \\
\hline \multicolumn{7}{|l|}{ Smokes cigarettes } \\
\hline SNNP: Southern Nation & ty and & Peoples & & & & \\
\hline
\end{tabular}




\begin{tabular}{|c|c|c|c|c|c|c|}
\hline \multirow[t]{2}{*}{ Variables } & \multicolumn{2}{|c|}{ Physical violence } & \multicolumn{2}{|c|}{$\begin{array}{l}\text { Emotional } \\
\text { Violence }\end{array}$} & \multicolumn{2}{|c|}{ Sexual Violence } \\
\hline & AOR & $95 \% \mathrm{Cl}$ & AOR & $95 \% \mathrm{Cl}$ & AOR & $95 \% \mathrm{Cl}$ \\
\hline No & Ref & Ref & Ref & Ref & Ref & Ref \\
\hline Yes & - & - & 2.59 & $\begin{array}{l}(1.18- \\
5.67)\end{array}$ & - & - \\
\hline \multicolumn{7}{|c|}{ Currently pregnant } \\
\hline No or unsure & Ref & Ref & Ref & Ref & Ref & Ref \\
\hline Yes & - & - & - & - & 1.62 & $\begin{array}{l}(1.06- \\
2.46)\end{array}$ \\
\hline \multicolumn{7}{|c|}{ Attitudes toward wife beating } \\
\hline Have no & Ref & Ref & Ref & Ref & Ref & Ref \\
\hline Have & 1.31 & $\begin{array}{l}(1.05- \\
1.65)\end{array}$ & - & - & - & - \\
\hline \multicolumn{7}{|c|}{$\begin{array}{l}\text { Women's decision-making } \\
\text { autonomy }\end{array}$} \\
\hline No & Ref & Ref & Ref & Ref & Ref & Ref \\
\hline Yes & 0.72 & $\begin{array}{l}(0.58- \\
0.89)\end{array}$ & 0.70 & $\begin{array}{l}(0.57- \\
0.86)\end{array}$ & 0.65 & $\begin{array}{l}(0.47- \\
0.89)\end{array}$ \\
\hline \multicolumn{7}{|c|}{ Husband/partner age } \\
\hline $30-34$ & Ref & Ref & Ref & Ref & Ref & Ref \\
\hline $15-19$ & - & - & 0.60 & $\begin{array}{l}(0.12- \\
3.03)\end{array}$ & - & - \\
\hline $20-24$ & - & - & 0.51 & $\begin{array}{l}(0.30- \\
0.87)\end{array}$ & - & - \\
\hline $25-29$ & - & - & 0.62 & $\begin{array}{l}(0.45- \\
0.87)\end{array}$ & - & - \\
\hline $35-39$ & - & - & 0.77 & $\begin{array}{l}(0.57- \\
1.05)\end{array}$ & - & - \\
\hline $40-44$ & - & - & 1.05 & $\begin{array}{l}(0.77- \\
1.44)\end{array}$ & - & - \\
\hline $45-49$ & - & - & 1.14 & $\begin{array}{l}(0.81- \\
1.61)\end{array}$ & - & - \\
\hline $50-54$ & - & - & 1.10 & $\begin{array}{l}(0.73- \\
1.65)\end{array}$ & - & - \\
\hline
\end{tabular}




\begin{tabular}{|c|c|c|c|c|c|c|}
\hline \multirow[t]{2}{*}{ Variables } & \multicolumn{2}{|c|}{ Physical violence } & \multicolumn{2}{|c|}{$\begin{array}{l}\text { Emotional } \\
\text { Violence }\end{array}$} & \multicolumn{2}{|c|}{ Sexual Violence } \\
\hline & AOR & $95 \% \mathrm{Cl}$ & AOR & $95 \% \mathrm{Cl}$ & AOR & $95 \% \mathrm{Cl}$ \\
\hline $55-59$ & - & - & 1.50 & $\begin{array}{l}(0.90- \\
0.50)\end{array}$ & - & - \\
\hline \multicolumn{7}{|c|}{ Husband/partner's education level } \\
\hline No education & Ref & Ref & Ref & Ref & Ref & Ref \\
\hline Primary & - & - & - & - & 1.13 & $\begin{array}{l}(0.81- \\
1.58)\end{array}$ \\
\hline Secondary & - & - & - & - & 0.71 & $\begin{array}{l}(0.40- \\
1.29)\end{array}$ \\
\hline Higher & - & - & - & - & 0.39 & $\begin{array}{l}(0.16- \\
0.93)\end{array}$ \\
\hline Don't know & - & - & - & - & 1.19 & $\begin{array}{l}(0.26- \\
5.42)\end{array}$ \\
\hline \multicolumn{7}{|c|}{ Husband/partner drinks alcohol } \\
\hline No & Ref & Ref & Ref & Ref & Ref & Ref \\
\hline Yes & 3.12 & $\begin{array}{l}(2.45- \\
3.97)\end{array}$ & 2.44 & $\begin{array}{l}(1.94- \\
3.08)\end{array}$ & 2.58 & $\begin{array}{l}(1.75- \\
3.80)\end{array}$ \\
\hline
\end{tabular}

\section{Discussion}

The purpose of this study was to find the spatial distribution of IPV and its determinant factors in Ethiopia. According the present study, there was a significant clustering of IPV in the study area and the highest IPV cluster was observed in Oromia, Somali and SNNP regions. This clustering of IPV in this area might be due to culture of a given society that did not recognize IPV as violation of human right rather accept and expect that wife beating is part of a normal union. This also might be low awareness and wrong attitude of husbands/partners toward negative consequent of women violence.

The present study documented that women age as a determinate factor of IPV. Relatively younger women were less likely to be physically abused as compared to older women. This finding is lined with household surveys in eight southern African countries (25) and Rwanda (26). This similar finding might be related to duration into the partnership of women's first IPV victimization. Most of time relationships of longer durations are more likely to involve a history of male-perpetrated violence (38).

This study revealed that women who were watching television were at low risk of physical violence as compared with their counterpart. This result might be due to positive effect of mass media like television 
on women's attitudes towards violence against women (39) and those women who such attitudes are less likely to be abused.

Cigarettes Smoking status is significantly associated with emotional violence. According to the present study, smoker women were more likely to be emotionally violated than their counterpart. Since the current study use secondary date that was conducted through community based cross-sectional study, it is difficult to sort out the causal ordering between cigarettes Smoking status and emotional violence. Some studies also documented that experiencing intimate partner violence (IPV) increases women's risk for cigarette smoking (40-43).

The results of this study suggested that pregnant women were more probable to be sexually violated than non-pregnant women. Such violence during pregnancy does not only affect the women's reproductive health, but also leads to adverse outcomes of maternal and child health (44-46).

In line with a study conducted in Uganda (47), the present study reported that women who have attitudes toward wife beating were more likely to be physically violated than their counterpart. The possible explanation for these results, women with attitudes supportive of IPV is more likely to be victims of IPV. Because of such attitudes, women may accept and expect that wife beating is part of a normal union.

Our finding demonstrated that women's decision-making autonomy were significantly associated with physical, emotional and sexual violence. The chances of physical, emotional and sexual violence were decreased among women who had decision making autonomy than their counterparts. This finding is in line with other studies that was conducted in Uganda (37), in Ghana (33) and in Peru (32). This consistency could be supported by a male-dominated marital power structure has been documented to be highly related with marital conflict and husband-to-wife violence (48). Women's decision-making autonomy is not only reduced the risk IPV but also increased the utilization of maternal service $(31,49)$.

In line with previous studies $(29,30)$, the current study reported that the greater wealth were protective against emotional violence. The possible explanation for these consistent results might be low household economic status is the reason for conflict between couples.

The current study also identified husband / partner related factors that associated with IPV such as husband /partner's age, educational level and alcohol drinking status. The present study reported that the likelihoods of emotional violence were decreased among young husband/partner's as compared with old husband/partners. A similar result has been found in Haiti (26). In concurrent with other studies $(27,28)$, the present study reported that the likelihoods of sexual violence were decreased among higher educated husband/partner than uneducated.

This study revealed that Husband/partner drinks alcohol as a predictor of IPV. According to current study drinker husband / partner were more likely to physically and sexually violate his wife/ partner than their counterparts. This result is in line with other studies that was conducted in Angola (36), in Uganda (34) and India (35). The possible explanation for these results alcohol uses directly disturbs mental and 
physical function, decreasing self-control and leaving individuals less capable of negotiating a nonviolent resolution to conflicts in relationships. Excessive drinking by one partner can worsen financial problems, child care difficulties, infidelity or other family stressors. This can lead to marital tension and conflict, increasing the risk of violence occurring between partners.

The primary strength of the current study was using large population-based data with a large sample size, which is representative at national and regional levels, so it can be generalized to all women in reproductive age group in Ethiopia. The joint use ArcGIS and Sat Scan statistical tests facilitated to identify similar and statistically significant area with high IPV (hot spot area). Furthermore, it used multivariate analysis (GSEM) to determine factors associated with three domains of IVP simultaneously.

However, the finding of this study interpreted with some limitation. First, the location of data values was displaced up to 2 kilometers for urban and up to 5 kilometers for rural areas to ensure respondent confidentiality, thus, this was the challenge to know the exact cases' location. Since EDHS was conducted using cross-sectional study design, it is difficult to sort out the causal ordering. Recall bias may be the other impediment for this study as EDHS was a questionnaire-based survey and relied on the memory of the respondents.

\section{Conclusion}

There was a significant clustering of IPV in the study area and the highest IPV cluster was observed in Oromia, Somali and SNNP regions. Being richest, watching television, not having attitudes toward wife beating, women's decision autonomy and husband's/partner's high education and non-alcohol drinker status were negatively associated with IPV. The likelihood of IPV was also increased among smoker and pregnant women. We recommend that improving the economic status of the household and power of women's decision autonomy, increasing community awareness about the consequences of IPV with particular emphasis in Oromia, Somali and SNNP regions.

\section{Abbreviations}

CSA

Central Statistics Agency; EAs:Enumeration Areas; EDHS:Ethiopian Demographic and Health Survey; GSEM:Generalized Structural Equation Model; IPV:Intimate partner violence; SNNP:Southern Nation and Nationality and Peoples; WHO:World Health Organization

\section{Declarations}

Ethics approval and consent to participate

The study doesn't include the collection of information from subjects. Consent to participate is not applicable. We sent a one-page proposal abstract of the study to the DHS program office. They gave permission to access the data with reference number of 142467. 
Consent for publication

Not applicable.

Availability of data and materials

All necessary information's and supplementary materials were included with in the manuscript.

Competing interest

All authors declare no conflicts of interest.

Funding

No fund was received for this work.

Authors' contributions

Conception of the work, design of the work, acquisition of data, analysis and interpretation of data was done by BS. Data curation, drafting the article, revising it critically for intellectual content, validation and final approval of the version to be published was done by BS, SA and AB. All authors read and approved the final manuscript

\section{References}

1. Organization WH. Responding to intimate partner violence and sexual violence against women: WHO clinical and policy guidelines: World Health Organization; 2013.

2. Organization WH. Researching violence against women: Practical guidelines for researchers and activists: World Health Organization; 2005.

3. Butchart A, Garcia-Moreno C, Mikton C. Preventing intimate partner and sexual violence against women: taking action and generating evidence. 2010.

4. Krug EG, Mercy JA, Dahlberg LL, Zwi AB. The world report on violence and health. The lancet. 2002;360(9339):1083-8.

5. Scribano PV, Stevens J, Kaizar E, Team N-IR. The effects of intimate partner violence before, during, and after pregnancy in nurse visited first time mothers. Matern Child Health J. 2013;17(2):307-18.

6. Okenwa L, Lawoko S, Jansson B. Contraception, reproductive health and pregnancy outcomes among women exposed to intimate partner violence in Nigeria. The European Journal of Contraception Reproductive Health Care. 2011;16(1):18-25.

7. Sarkar $\mathrm{N}$. The impact of intimate partner violence on women's reproductive health and pregnancy outcome. J Obstet Gynaecol. 2008;28(3):266-71.

8. Emenike E, Lawoko S, Dalal K. Intimate partner violence and reproductive health of women in Kenya. International nursing review. 2008;55(1):97-102. 
9. Golding JM. Intimate partner violence as a risk factor for mental disorders: A meta-analysis. Journal of family violence. 1999;14(2):99-132.

10. Campbell JC. Health consequences of intimate partner violence. The lancet. 2002;359(9314):13316.

11. Campbell JC, Baty M, Ghandour RM, Stockman JK, Francisco L, Wagman J. The intersection of intimate partner violence against women and HIV/AIDS: a review. International Journal of Injury Control Safety Promotion. 2008;15(4):221-31.

12. García-Moreno C, Pallitto C, Devries K, Stöckl H, Watts C, Abrahams N. Global and regional estimates of violence against women: prevalence and health effects of intimate partner violence and nonpartner sexual violence: World Health Organization; 2013.

13. Semahegn A, Mengistie B. Domestic violence against women and associated factors in Ethiopia; systematic review. Reproductive health. 2015;12(1):78.

14. Agency CS, ICF. Ethiopia Demographic and Health Survey 2016: Key Indicators Report. Addis Ababa, Ethiopia, and Rockville, Maryland, USA. CSA and ICF. 2016.

15. Lailulo YA, Susuman AS, Blignaut R. Correlates of gender characteristics, health and empowerment of women in Ethiopia. BMC Womens Health. 2015;15(1):116.

16. Yigzaw T, Yibric A, Kebede Y. Domestic violence around Gondar in northwest Ethiopia. Ethiopian Journal of Health Development. 2004;18(3):133-9.

17. Semahegn A, Belachew T, Abdulahi M. Domestic violence and its predictors among married women in reproductive age in Fagitalekoma Woreda, Awi zone, Amhara regional state, North Western Ethiopia. Reproductive health. 2013;10(1):63.

18. Feseha G, Gerbaba M. Intimate partner physical violence among women in Shimelba refugee camp, northern Ethiopia. BMC Public Health. 2012;12(1):125.

19. Deribew A. Magnitude and risk factors of intimate partner violence against women in Agaro Town, Southwest Ethiopia. Ethiopian Journal of Health Sciences. 2007;17(2):99-108.

20. Abeya SG, Afework MF, Yalew AW. Intimate partner violence against women in western Ethiopia: prevalence, patterns, and associated factors. BMC Public Health. 2011;11(1):913.

21. Deyessa N, Berhane Y, Alem A, Ellsberg M, Emmelin M, Hogberg U, et al. Intimate partner violence and depression among women in rural Ethiopia: a cross-sectional study. Clinical practice epidemiology in mental health. 2009;5(1):8.

22. United Nations Department of Economics and Social Affairs PD United Nations Department of Economics and Social Affairs PD. Worldometer:; [cited 2020 6/2/2020]. Available from: https://www.worldometers.info/world-population/ethiopia-population/.

23. Straus MA, Douglas EM. A short form of the Revised Conflict Tactics Scales, and typologies for severity and mutuality. Violence victims. 2004;19(5):507-20.

24. 24. International journal of environmental research and public health. 
25. Andersson N, Ho-Foster A, Mitchell S, Scheepers E, Goldstein S. Risk factors for domestic physical violence: national cross-sectional household surveys in eight southern African countries. BMC Womens Health. 2007;7(1):11.

26. Hindin MJ, Sunita Kishor. and Donna L. Ansara. Intimate Partner Violence among Couples in 10 DHS Countries: Predictors and Health Outcomes. Calverton, Maryland, USA:: 2008 .DHS Analytical Studies.

27. Abrahams N, Jewkes R, Laubscher R, Hoffman M. Intimate partner violence: prevalence and risk factors for men in Cape Town, South Africa. Violence victims. 2006;21(2):247-64.

28. Flake DF. Individual, family, and community risk markers for domestic violence in Peru. Violence Against Women. 2005;11(3):353-73.

29. Jeyaseelan L, Kumar S, Neelakantan N, Peedicayil A, Pillai R, Duvvury N. Physical spousal violence against women in India: some risk factors. J Biosoc Sci. 2007;39(5):657-70.

30. Luke N, Schuler SR, Mai BT, Vu Thien P, Minh TH. Exploring couple attributes and attitudes and marital violence in Vietnam. Violence Against Women. 2007;13(1):5-27.

31. Sripad P, Warren CE, Hindin MJ, Karra M. Assessing the role of women's autonomy and acceptability of intimate-partner violence in maternal health-care utilization in 63 low- and middle-income countries. Int J Epidemiol. 2019;48(5):1580-92.

32. Svec J, Andic T. Cooperative Decision-Making and Intimate Partner Violence in Peru. Popul Dev Rev. 2018;44(1):63-85.

33. Tenkorang EY. Women's Autonomy and Intimate Partner Violence in Ghana. International perspectives on sexual reproductive health. 2018;44(2):51-61.

34. Tumwesigye NM, Kyomuhendo GB, Greenfield TK, Wanyenze RK. Problem drinking and physical intimate partner violence against women: evidence from a national survey in Uganda. BMC Public Health. 2012;12:399.

35. Wagman JA, Donta B, Ritter J, Naik DD, Nair S, Saggurti N, et al. Husband's Alcohol Use, Intimate Partner Violence, and Family Maltreatment of Low-Income Postpartum Women in Mumbai, India. J Interpers Violence. 2018;33(14):2241-67.

36. Yaya SGB. Alcohol drinking by husbands/partners is associated with higher intimate partner violence against women in Angola. Safety. 2019;5(1):5.

37. Zegenhagen S, Ranganathan M, Buller AM. Household decision-making and its association with intimate partner violence: Examining differences in men's and women's perceptions in Uganda. SSM population health. 2019;8:100442.

38. Sutton D, Dawson M. Differentiating characteristics of intimate partner violence: do relationship status, state, and duration matter? Journal of interpersonal violence. 2018:0886260518795501.

39. Jesmin SS, Amin I. Impact of the Mass Media in Changing Attitudes Towards Violence Against Women in Bangladesh: Findings from a National Survey. Journal of Family Violence. 2017;32(5):525-34. 
40. Caleyachetty R, Echouffo-Tcheugui JB, Stephenson R, Muennig P. Intimate partner violence and current tobacco smoking in low- to middle-income countries: Individual participant meta-analysis of 231,892 women of reproductive age. Glob Public Health. 2014;9(5):570-8.

41. Jun HJ, Rich-Edwards JW, Boynton-Jarrett R, Wright RJ. Intimate partner violence and cigarette smoking: association between smoking risk and psychological abuse with and without cooccurrence of physical and sexual abuse. American journal of public health. 2008;98(3):527-35.

42. Lemon SC, Verhoek-OftedahI W, Donnelly EF. Preventive healthcare use, smoking, and alcohol use among Rhode Island women experiencing intimate partner violence. J Womens Health Gend Based Med. 2002;11(6):555-62.

43. Stueve A, O'Donnell L. Continued smoking and smoking cessation among urban young adult women: findings from the Reach for Health longitudinal study. American journal of public health. 2007;97(8):1408-11.

44. Berhanie E, Gebregziabher D, Berihu H, Gerezgiher A, Kidane G. Intimate partner violence during pregnancy and adverse birth outcomes: a case-control study. Reprod Health. 2019;16(1):22.

45. Yu H, Jiang X, Bao W, Xu G, Yang R, Shen M. Association of intimate partner violence during pregnancy, prenatal depression, and adverse birth outcomes in Wuhan, China. BMC Pregnancy Childbirth. 2018;18(1):469.

46. Demelash H, Nigatu D, Gashaw K. A Case-Control Study on Intimate Partner Violence during Pregnancy and Low Birth Weight, Southeast Ethiopia. Obstetrics Gynecology International. 2015;2015:394875.

47. Speizer IS. Intimate partner violence attitudes and experience among women and men in Uganda. J Interpers Violence. 2010;25(7):1224-41.

48. Coleman DH, Straus MA. Marital power, conflict, and violence in a nationally representative sample of American couples. Violence victims. 1986;1(2):141-57.

49. Gautam S, Jeong HS. The Role of Women's Autonomy and Experience of Intimate Partner Violence as a Predictor of Maternal Healthcare Service Utilization in Nepal. 2019;16(5).

\section{Figures}




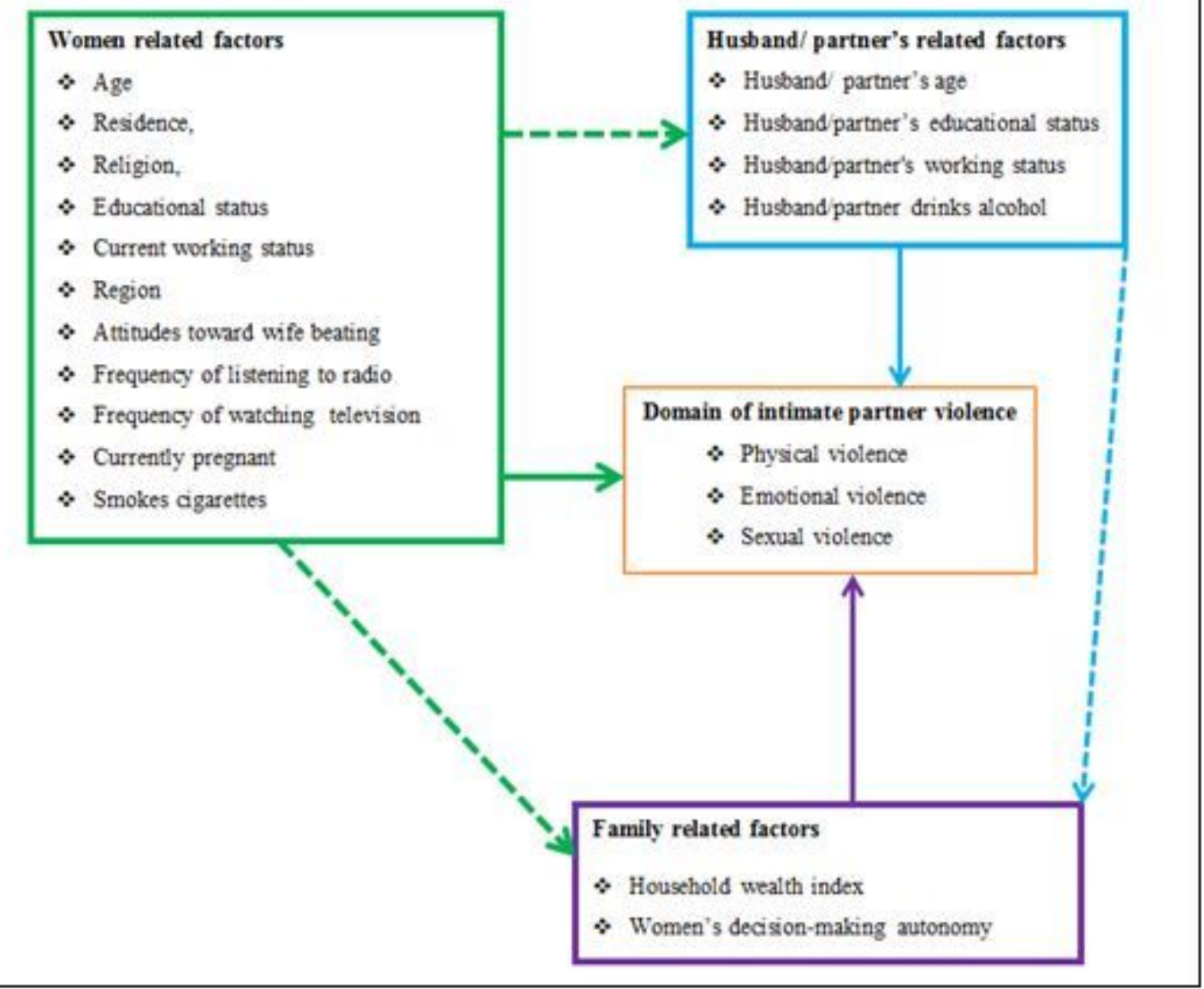

\section{Figure 1}

Hypothesized model for spatial distribution and determinant factors of intimate partner violence among reproductive age group women in Ethiopia. 
Moran's Index: 0.089642

z-score: 5.452609

p-value: 0.000000

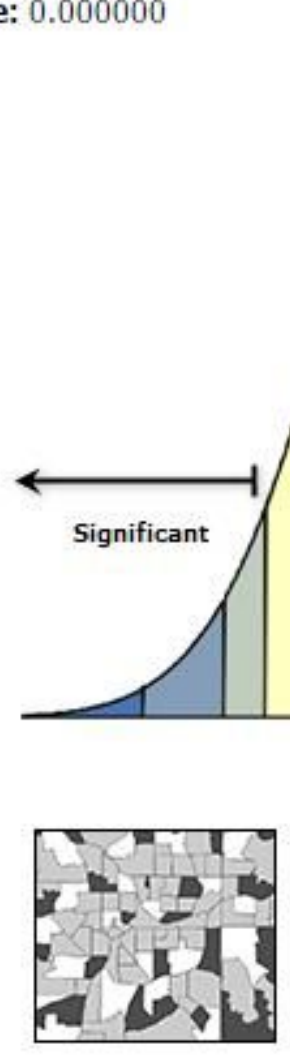

Dispersed

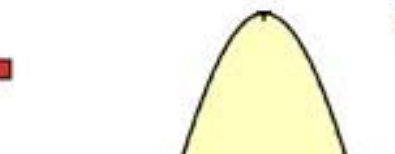

Significance Level ( $p$-value)

0.01

0.05

0.10

0.10

0.10

0.01
Critical Value

(z-score)

$\square<-2.58$

$-2.58--1.96$

$-1.96--1.65$

$-1.65-1.65$

1.65-1.96

ㄱ. $1.96-2.58$

$>2.58$

\section{Figure 2}

Spatial autocorrelation analysis of intimate partner violence in Ethiopia, 2016 


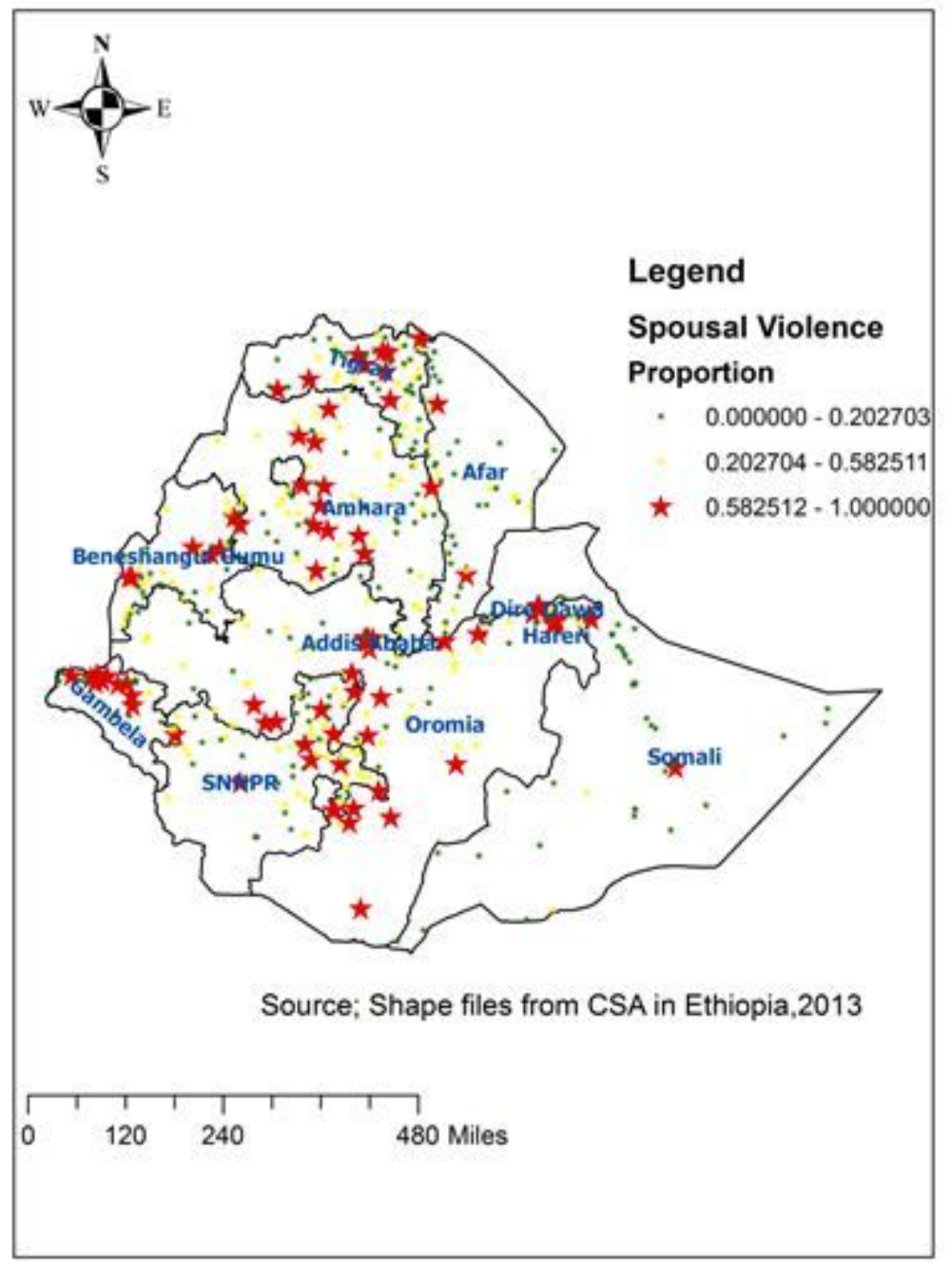

Figure 3

Spatial distribution of intimate partner violence across regions in Ethiopia, 2016 


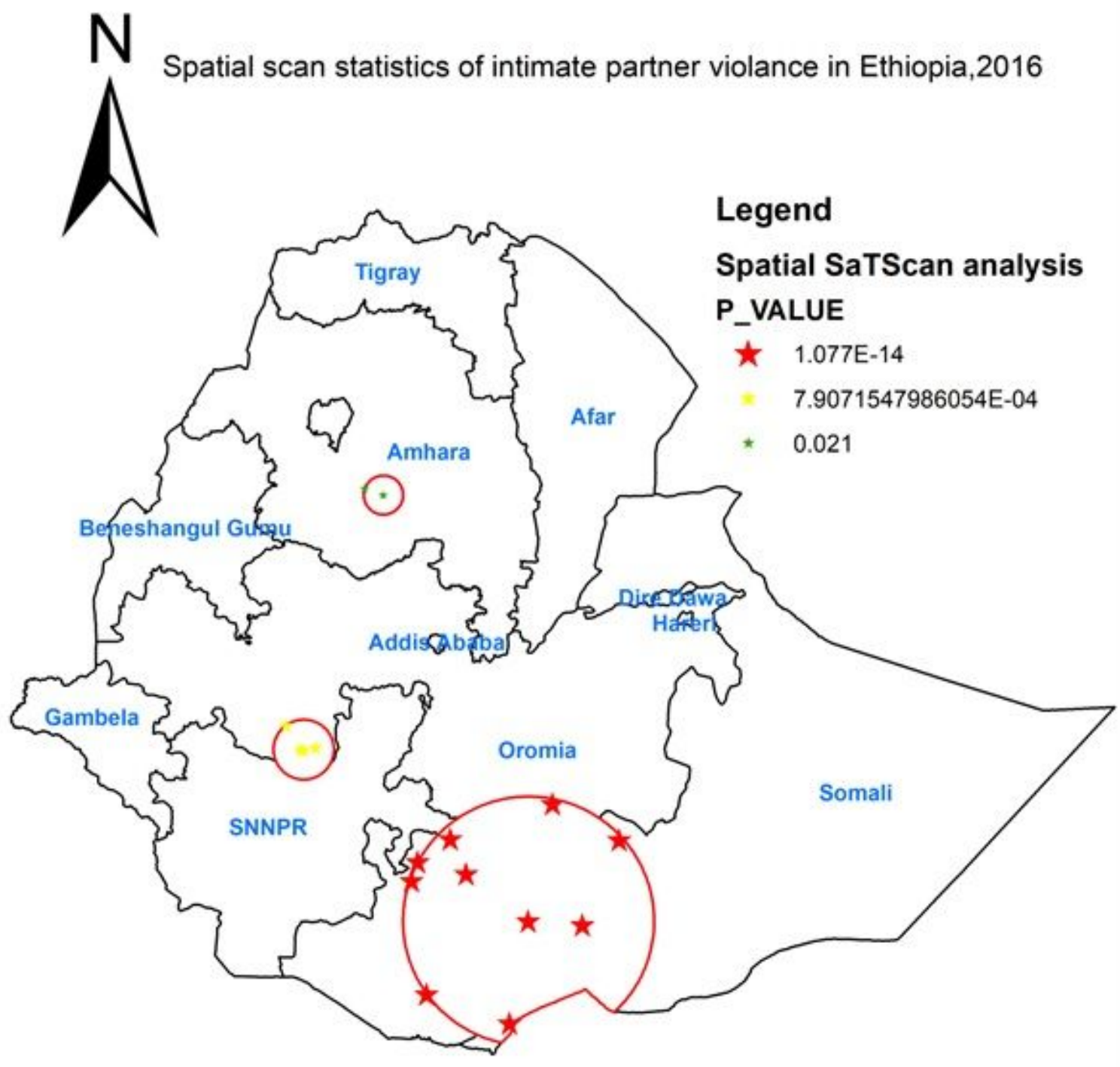

Source:Shape file from CSA in Ethiopia,2013

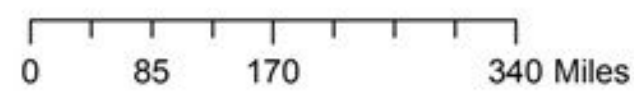

\section{Figure 4}

Primary and secondary clusters of health insurance coverage among women across regions in Ethiopia, 2016 


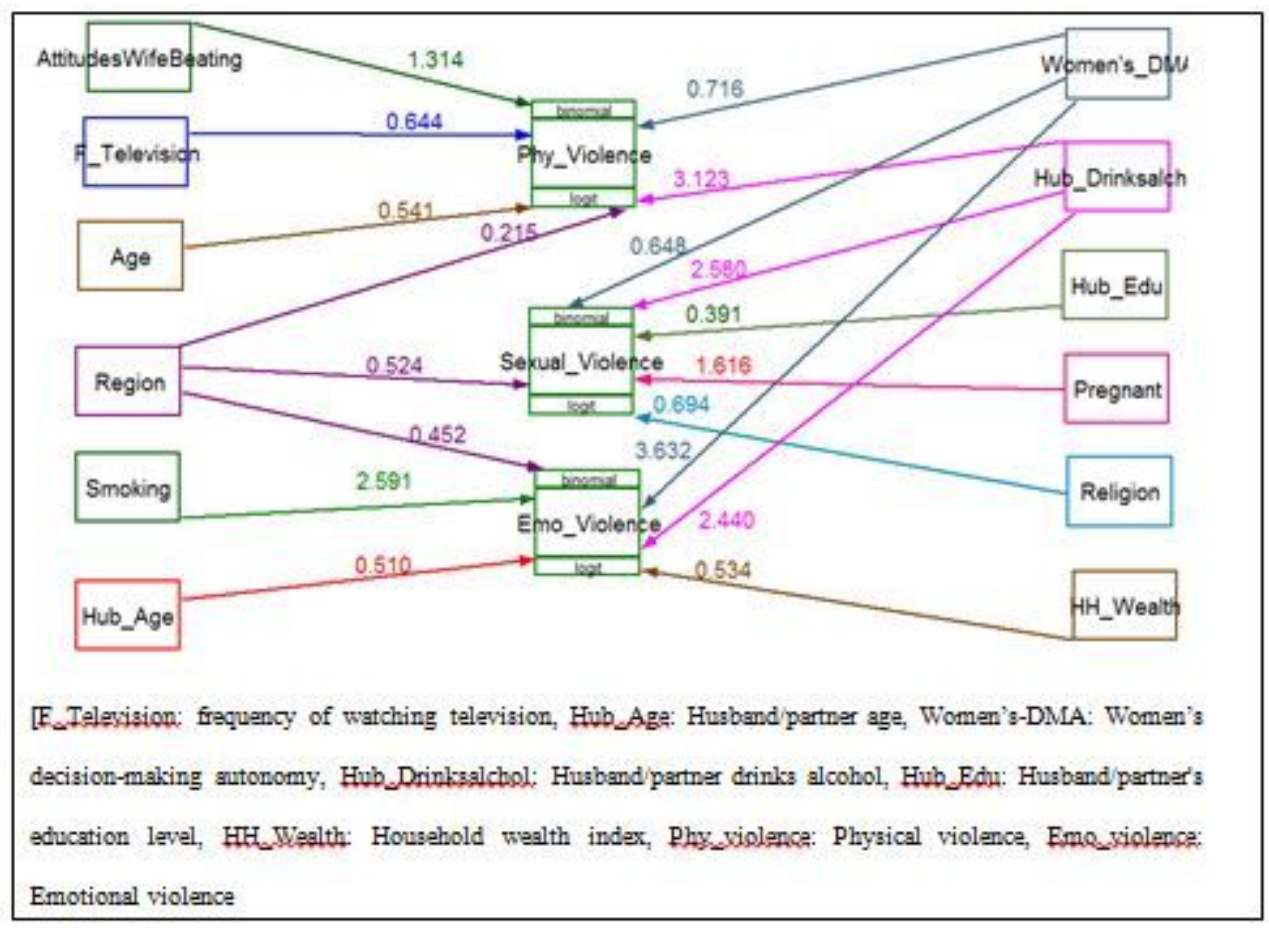

\section{Figure 5}

GSEM for determinant factors of intimate partner violence among reproductive age group women in Ethiopia, 2016. 